\title{
A Framework for using Machine Learning to Support Qualitative Data Coding
}

\author{
Peter Baumgartner ${ }^{1}$ | Amanda Smith ${ }^{2}$ | Murrey \\ Olmsted $^{3}$ | Dawn Ohse ${ }^{4}$
}

${ }^{1}$ RTI International

${ }^{2}$ RTI International

${ }^{3}$ RTI International

${ }^{4}$ RTI International

Correspondence

Peter Baumgartner, M.S., Center for Data Science, RTI International, Durham, NC, 27709, United States of America

Email: pbaumgartner@rti.org

Present address

RTI International, Durham, NC, 27709, United

States of America

Funding information
Open-ended survey questions provide qualitative data that are useful for a multitude of reasons. However, qualitative data analysis is labor intensive, and researchers often lack the needed time and resources resulting in underutilization of qualitative data. In attempting to address these issues, we looked to machine learning and recent advances in language models and transfer learning to assist in qualitative coding of responses. We trained a machine learning model following the BERT architecture to predict thematic codes that were then adjudicated by human coders. Results suggest this is a promising approach that can be used to support traditional coding methods and has the potential to alleviate some of the burden associated with qualitative data analysis.

Keywords - Qualitative Coding, Transfer Learning, Machine Learning, Natural Language Processing, Survey Statistics

\section{INTRODUCTION}

\section{Survey Background}

\section{Open-ended / Free-text Survey Questions}

Researchers ask open-ended questions (i.e., questions that do not include defined response categories) on surveys for a variety of reasons. These data can help survey researchers add depth and nuance to quantitative findings and can also be used to better understand phenomena before drafting future questions. For instance, if little is known about the audience's attitudes or opinions on a specific topic or issue, asking an open-ended question will provide participants with an opportunity to express themselves without limiting their response. The responses to these questions frequently reveal both the thought processes that participants are engaging in as well as more candid opinions than can typically be expressed using standard closed-ended questions (Lazarsfeld, 
1944; Reja et al., 2003). Another advantage of using open-ended questions is that they can identify new information about participants attitudes and opinions. For example, asking employees what changes would have the biggest impact on their satisfaction with work may identify both positive and negative aspects of their work life that would otherwise not be known to researchers allowing possible action to take place to address these issues (Tourangeau et al., 2000).

While open-ended questions offer some distinct advantages, they also present some challenges. For instance, open-ended questions require that participants are literate and able to express themselves in writing. This can be a challenge as not all survey audiences are literate and able to provide a cogent response to an open-ended question. Even if respondents are literate, interpreting the content, meaning, and sentiment of text responses can be difficult at times (Tourangeau et al., 2000). This is partly due to the mechanics of the writing and how well participants are able to express themselves, but also is due to the fact that text data needs to be interpreted by the researcher. This interpretation is inherently subjective and so it can be very inconsistently interpreted by different users of the data. For this reason, many researchers turn to coding as a way of attempting to standardize analyses of the text data from the open-ended questions (Braun and Clarke, 2006). And finally, another concern with using open-ended questions comes from past research which shows that participants frequently see these items as being more burdensome and therefore are more likely to skip over these questions leading to more item non-response (Denscombe, 2009).

Likely one of the biggest challenges, however, with including open-ended responses on surveys is that it is simply very time consuming to analyze qualitative data. When time and resources are limited, researchers are often faced with the decision to either forgo open-ended questions altogether, and the potential insights they can provide, or decide to only do a cursory analysis the data that could potentially result in key insights be overlooked.

There are certainly both advantages and disadvantages to consider when using open-ended survey questions on surveys. However, while challenging, there is no disputing that these questions often provide valuable information that would otherwise be difficult to obtain in a closed-ended item (Reja et al., 2003).

\section{| Qualitative Coding \& Classification}

Qualitative data analysis of text data collected from open-end survey responses typically consists of some form of data coding. There are various methods for coding qualitative data, but the ultimate goal is to assign labels (i.e., codes) to chunks of data (e.g., words, groups of words, whole sentences etc.) in an effort to categorize or summarize the data, uncover patterns or themes, and ultimately develop theories that work to explain observations in the data (Miles et al., 2013).

Qualitative coding can begin with a set of predetermined codes (i.e., deductive, a priori) based on an existing theoretical framework or prior knowledge, or the coding process and codes may be guided by the data itself. The latter approach, most often associated with Grounded Theory as codes are "grounded" in the data, is frequently used to explore data where there is limited prior knowledge about the topic being examined (Charmaz, 2006). This type of coding consists of at minimum two phases initial/open coding and focused coding (Charmaz, 2006). During the initial, open coding phase coders review the data carefully to construct labels (i.e., codes) that work to break down and classify data that are conceptually similar(Charmaz, 2006; Corbin and Strauss, 1990). The second phase consists of using initial codes to sort through and re-examine larger segments of data, while also determining which codes "make the most analytic sense to categorize your data incisively and completely" (Charmaz, 2006). This process is often iterative and involves some level of refining and revising initial codes. In short, this approach typically consists of an initial read of the data to understand and categorize, then a process of reviewing and re-reviewing the data and refining codes so they best conceptualize what is being observed in the data.

As is evident from the above, qualitative data analysis is labor intensive. Some efforts to help reduce the burden of qualitative analysis start during the question development process. For instance, open-ended questions on web surveys may restrict character counts, and researchers may craft more direct questions to orient the respondent, which makes it a somewhat easier to control the variation and volume of data received. However, this has potential drawbacks, such as narrowing focus to the extent valuable 
data are missed, and ultimately still results in text data that need to be organized and analyzed.

Another limitation is dealing with inherent inconsistencies in human coding. At the most basic level, even with consistent and clear code definitions, humans can make mistakes in coding the data, or introduce variability in how codes are applied. Lack of agreement between coders can result in low inter-rater reliability, either due either to errors in human coding or subjective differences in interpretation of code definitions and application rules.

Earlier "automated" methods, such as word counts using qualitative data analysis software (QDAS), sought to lessen the burden of coding. However, these methods are simplistic and typically provide only a surface understanding of the data, often failing to capture nuance, sentiment, and context that is key to understanding qualitative data. For instance, it is difficult to pull larger themes from simple word counts, which is key to understanding phenomena and addressing research questions. And even more recent advances in automation using QDAS software, such as keyword coding or auto coding based on existing coding patterns, have many limitations and often fall short of the robustness that can be accomplished with human coding. As such, "manual" human coding has historically been the best method for capturing the true essence of qualitative data. However, advances in machine learning have opened up new computational approaches to working with text data that are more robust than the automation methods available in QDAS platforms.

\section{Computational Approaches for Text Classification}

Classic computational approaches to analyzing text data arose from content analysis methods for longer texts. These approaches relied on dictionaries, which were mappings of words to categories. Analysis proceeded by counting up the occurrences of words or phrases that were assigned into categories, then testing hypothesis about differences in category frequency across texts. The first mainstream computational analysis tool was the General Inquirer (GI), which mapped words to categories such as valence (positive / negative), Osgood's semantic dimensions (evaluation, potency, activity), and several other categories. The most recent version of the General Inquirer has 182 categories and is available online (Stone and Hurwitz, 2012).

The General Inquirer manual contains example applications for survey research and is explicit about the limitations of the approach (Stone et al., 1966). Using the GI for surveys required significant development time, particularly if there was no overlap in existing conceptual categories since this required the development of new categories and associated definitions, rules, and words. In addition, alignment on concepts or theories represented by the categories was often difficult. The online documentation states that categories were "developed for social-science content-analysis research applications, not for text archiving, automatic text routing, automatic text classifying, or other natural-language processing objectives, although they may be relevant to some of them" and that "many categories were initially created to represent social-science concepts of several grand theories that were prominent at the time the system was first developed." (Stone and Hurwitz, 2012)

From a technical perspective, the dictionary-based approach as exemplified by the GI lacks precision. For example, the positive valence expressed in "The movie was good" and "The movie was amazing" is different, however, a dictionary-based approach would give equal weight to the words "good" and "amazing". While there are supplemental calculations to weighting words one could make using a dictionary-based approach, those solutions are untenable for larger dictionaries and more complex linguistic patterns.

Statistical approaches to analyzing text with natural language processing became popular as both computational power and data availability increased. Classic machine learning emerged as a paradigm for building text classification and categorization systems by combining large text data sources and algorithms (i.e., models) such as logistic regression or support vector machines (Flach, 2012). In this paradigm, referred to as supervised learning, models learn statistical relationships between the terms in a text and a topic, outcome, or labels of interest. Concretely, instead of explicitly specifying rules-e.g., the presence of the word "good" indicates positive valence-that relationship is learned from many examples of positive sentences that contain the word "good". These systems also learn weights associated with each term, that is, how much the word "good" might contribute to 
the positive classification of a text. Once a machine learning model has learned these statistical relationships, the model can be applied at a larger scale to "predict" the positive or negative valence, or any classification scheme it has learned, to new texts.

While classical machine learning allows for scalable and more precise classification, it also comes with limitations. Firstly, it requires a significant amount of data for the training process. In addition, the data it learns from (training data) must be labeled accurately: any bias or errors from labeling the data are propagated through the machine learning model and impact the performance. Additional potential issues are evident when we decompose a machine learning system into two parts: the vocabulary that the model has learned and the associated weights for terms in that vocabulary. The vocabulary the model has learned is composed only of words that the model has seen in the training data. When applying the machine learning system to new texts, previously unseen terms do not get used. In essence, classic machine learning is a more precise and scalable statistical pattern recognition system, but it has not learned any semantic information from the text.

As computational approaches advanced and even larger text corpora became available, researchers used unsupervised learning approaches aimed at capturing semantic information within texts. In these situations, labels for texts do not exist. Instead, unsupervised learning captures and represents patterns inherent in data (Flach, 2012). A common unsupervised learning approach is generating word embeddings. Word embeddings are a mapping of a word to an embedding (a vector, or list of numbers), such that words that share common contexts are close to each other in the embedding space. Specifically, since the words "good" and "amazing" are often used in similar contexts, they should have embeddings near each other in the embedding space. Said differently, the distance between "good" and "amazing" in the embedding space is smaller than the distance between "good" and an arbitrary word such as "paper".

In 2013, an algorithm named word2vec was released that allowed for training word embedding models at a speed and scale not previously possible (Mikolov et al., 2013). The increased speed of word2vec combined with larger collections of text meant that word embedding models could be generated for whole languages with large vocabularies. Word embedding models addressed two of the previously mentioned problems with classic machine learning: the absence of important words in the training data and the lack of a semantic representation of those words. Perhaps most importantly during this era researchers released trained word-embedding models and made them available for public use, enabling researchers and practitioners without access to the resources required for training a model to use and adapt word embedding models.

Despite the significant progress in machine learning, two related linguistic issues and one machine learning issue remain. The linguistic issues are sentence structure and homography. These issues are illustrated with the word bank in the following two sentences:

- I went to the bank of the river.

- I went to the bank to deposit a check.

As this example illustrates, the context in which a word is used is important. With standard word embeddings, the embedding for the word bank inherits information from every context in which it is used. In this example it would represent both the geographic definition and the financial definition.

The machine learning issue that remains is the large amount of data required to train models. Several thousand documents are still required to train a machine learning system in order for it to generalize enough to perform well on future cases (Crowston et al., 2010; Abdelwahab et al., 2015). As an analogy, imagine if you had to re-learn how to read entirely every time you picked up a new book - that is how classic machine learning systems operate.

To continue the analogy: humans who know how to read remember the process of reading and can generalize it to new books. They might have to fine-tune their reading style between a textbook and a novella, but the general skill of reading allows them to handle both types of texts. The machine learning paradigm of transfer learning aligns closer to this model.

When transfer learning is used for text, a large language model is trained on a massive corpus of text (Devlin et al., 2018; 
Howard and Ruder, 2018). This language model captures a fuller semantic representation of how a language works, including the complexities of sentence structure. These large language models are also able to generate contextual embeddings for words within sentences. In the two example sentences containing bank, a language model generates different embeddings for all senses of bank that represent the different contexts in which those homographs are used (Wiedemann et al., 2019).

Building the language model is only the first part of the process. Because of their computational and data requirements, these language models are typically trained by research teams and then published online so that future use of these models doesn't require retraining.

As applied, these language models are fine-tuned to specific tasks like text classification. In addition to handling the linguistic issue of contextual-word use, language models and transfer learning also reduce the amount of training data required to build a text classification system. In some situations, comparable performance to classic machine learning models is obtained with transfer learning models using many fewer examples (Howard and Ruder, 2018). Pragmatically, transfer learning makes it possible to build a text classification system with the amount of data typically collected in a survey context.

\section{METHODS \& DATA}

\section{RTI Employee Survey Background}

The RTI Employee Survey is a comprehensive assessment of employee experience including topics such as engagement, workplace culture, benefits, and leadership effectiveness. The survey was administered to more than 4,500 RTI employees in 2018 and 2019 and was available in 6 languages: English, French, Spanish, Bahasa Indonesian, Arabic, and Nepali. Both the 2018 and 2019 surveys included the following open-ended question at the end of the survey: What is the most important change RTI could make to improve your experience working at RTI? In both years there were almost 3,000 responses submitted to this question.

The RTI executive leadership needed to quickly understand the opportunities for improvement to the employee experience and to possible follow-up actions accordingly. Therefore, thematic coding was used to categorize comments into themes to provide a sense of the depth and breadth of themes for the comments.

\section{Cleaning and Translations}

All comments in a language other than English were translated using Google Translate into English so that coding could be done in a common language. A team of researchers (3 in 2018 and 6 in 2019) read all comments and flagged them for review if they contained names or enough detail that the employee providing the comment could potentially be identified. After review by the research team and Human Resources, 25 and 27 responses in 2018 and 2019, respectively, were revised or redacted to remove names or identifying details.

\section{Code Creation, Calibration, and Coding}

To develop an initial list of codes (i.e., themes) in 2018, three researchers read through the comments and then met to develop an initial list of codes (i.e., themes). To calibrate the codes, all three team members coded the same set of 150 comments and then met to review and discuss coding discrepancies. Comments could contain multiple themes, and thus each comment could be ascribed more than one code. Through this initial coding, additional codes were created as needed and a set of code definitions was finalized. The remaining comments were then divided up between the three researchers and coded separately using the final list of 26 codes and code definitions. Two of the 26 codes were not used for this task due to a low number of responses assigned 
that code and concerns about accuracy in those circumstances. In all, 24 codes were evaluated for this task.

\section{Use of Codes}

The code assignments are used by stakeholders to filter down the set of open-ended responses to the area of interest. In addition, the relative distribution of codes is analyzed between various groups of respondents. The frequency of codes is not used to analyze the relative importance of the issues to the organization for several reasons. First, the codes are not equivalent in scope: some codes cover broader and more abstract ideas that we would expect more responses to fall under. Second, the codes do not directly measure the sentiment of the response to the question, with the exclusion of the "Satisfaction" code. This means a response coded as "Development" and "Satisfaction" could indicate that the respondent expressed satisfaction with Development, or that they mentioned issues within the Development topic and their Satisfaction distinctly.

\section{Code Prediction Methodology}

Due to the burden of developing and applying codes, and the improvements in machine learning driven from transfer learning, we decided to evaluate a new method of coding prediction for the present study. This process involved training a predictive model based on the 2018 survey responses and associated codes, then using this model to predict which codes should be applied to new 2019 survey responses to the same question. The goal was to evaluate how well machine learning could match the codes to the data, and where possible, identify potential matches that the coders might not be aware of.

The new method involves training a predictive model on the 2018 survey responses and associated codes, which enables us to predict which codes should be applied to new 2019 survey responses. The goal was to evaluate how well machine learning could match the codes to the data, and where possible, identify potential matches that the coders might not be aware of.

To augment the dataset of survey responses, most of the 2018 responses went through a process of backtranslation—being translated to Spanish and back to English—to augment the training dataset with more examples. A handful $(\mathrm{n}=15)$ of responses were not able to be translated.

In addition, the first 150 responses of the 2019 survey were coded by humans initially to refine the coding scheme. These 150 examples were also included in the training data.

The specific model used was the bert-base-uncased, with parameters of maximum sequence length of 128 , learning rate of 5e-5, and trained for 3 epochs. The data used and process is outlined in Figure 1.

The new coding process works as follows:

1. Generate a prediction: Using the trained model, for each text response, generate a prediction whether each code should be applied for that response. The prediction is a number between 0 and 1 .

2. Convert to binary classification: If the model generates a predicted probability of a code being applied over 0.5 , signify a positive prediction with an " $\mathrm{x}$ "

3. Apply across all responses: This process results in a spreadsheet containing rows for each response, columns for each code, and an " $\mathrm{x}$ " in the cell if the code was predicted for that response.

With this spreadsheet, the responses were split up into 6 even groups for a team of coders to review and adjudicate. The coders adjudicated the predictions by reviewing each response and taking the following actions:

1. If the code was predicted, but shouldn't be applied (false positive): replace the previous " $x$ " cell value with a " $D$ "

2. If the code was not predicted, but should be applied (false negative): replace the cell value of "(blank)" with an "R" 


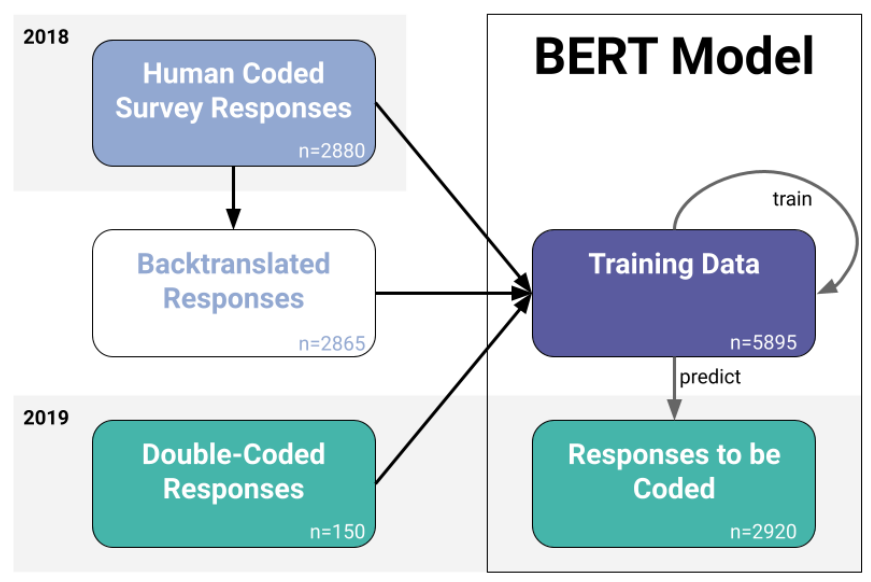

FIGURE 1 An outline of the data and pipeline used for the model training and auto-coding process.

3. If the application of a code was correctly predicted (true positive): do nothing - the cell remains an " $x$ "

4. If the absence of a code was correctly predicted (true negative): do nothing - the cell remains "(blank)"

\section{Analysis \& Findings}

To analyze the performance of the model, we compare the set of codes predicted by the model to the set of codes after final human review. The code set predicted by the model matched the final adjudication for $67.5 \%$ of responses. The performance on this metric (also called subset accuracy or exact match) is strongly related to the number of codes we attempt to predict. When evaluating predictions for the top 5 most frequent codes, that set of predictions matches human review $88.9 \%$ of the time. A summary of performance when only looking at the top $n$ codes, as measured by frequency in the training set, is in Figure 2.

The overall performance of the model can also be understood by evaluating the yes/no decision to apply each code to each response. Viewed this way, there are 70,080 unique yes/no decisions (2,920 responses $\times 24$ codes) for the 2019 survey responses. The final, human reviewed coding process resulted in 5761 positive (yes) code applications; while the model predicted a total of 4910 positive applications. Of those 5761 human applications of a code, 4695 of them (81.5\%) were predicted by the model. Of the 4910 model predictions, 215 of them were false positives: responses where the code was predicted but should not have been applied. As referred to in the machine learning literature, these two performance measures are the overall recall (81.5\%) and precision (95.6\%) of our model (Flach and Kull, 2015). A final measure that provides a summary of performance is the Matthews Correlation Coefficient (MCC or the phi coefficient in non-machine learning contexts), which was 0.87 (Chicco and Jurman, 2020).

An overall summary of model performance can be seen in Table 1, a $2 \times 2$ contingency table (confusion matrix) of actual and predicted codes. 


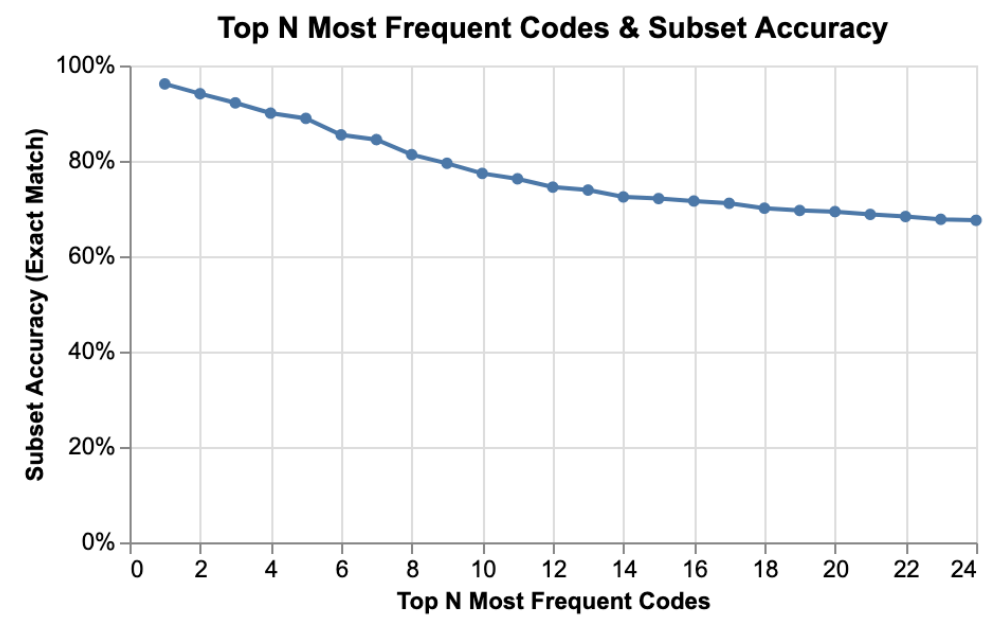

FIGURE 2 Subset accuracy for the most frequently used codes. Subset accuracy decreases when calculated on a larger set of codes.

Predicted

\begin{tabular}{|ccccc|}
\hline & & No & Yes & Total \\
\hline \multirow{2}{*}{ Actual } & No & 64104 & 215 & 64319 \\
& Yes & 1066 & 4695 & 5761 \\
\hline & Total & 65170 & 4910 & 70080 \\
\hline
\end{tabular}

TAB LE 1 Confusion matrix of actual (adjudicated) and predicted codes.

\section{Performance Per Code}

We also evaluated the model's performance on each of the 24 codes in the set by converting the problem to 24 binary (yes/no) classification tasks, the results of which are presented in Table 2. The mean code precision is 95\%, the mean code recall is $78 \%$, and the mean MCC is 0.85 . Overall, these results indicate that the model can effectively apply codes to the data with reasonable accuracy. The precision is higher than the recall, indicating the model did a better job of avoiding false negatives than missing false positive. These results are similar to performance on comparable text classification tasks using standard methods on benchmark datasets (Janani and Vijayarani, 2019; Tripathy et al., 2016).

There is a weak correlation $(\rho=0.34)$ between the number of training examples and the performance per code. This relationship is illustrated in Figure 3 below.

\section{DISCUSSION}

\section{Benefits}

The use of the model provided several benefits to the survey team. First, having human coders review and either confirm or adjudicate codes proved to be a simpler and more efficient task than following a "traditional" coding approach (i.e., reading all 


\begin{tabular}{|c|c|c|c|c|}
\hline Code & N Training & Precision & Recall & $\mathrm{MCC}$ \\
\hline Development & 839 & 97 & 91 & 0.91 \\
\hline Management & 430 & 96 & 90 & 0.92 \\
\hline Processes & 345 & 98 & 85 & 0.9 \\
\hline Communication & 325 & 96 & 80 & 0.86 \\
\hline Teamwork & 318 & 95 & 89 & 0.91 \\
\hline Fairness & 308 & 96 & 66 & 0.78 \\
\hline Compensation & 312 & 96 & 94 & 0.94 \\
\hline Strategy & 271 & 97 & 58 & 0.73 \\
\hline International & 261 & 94 & 81 & 0.86 \\
\hline Workload & 218 & 93 & 71 & 0.8 \\
\hline Recognition & 179 & 88 & 82 & 0.84 \\
\hline Satisfaction & 174 & 94 & 66 & 0.78 \\
\hline Morale & 162 & 97 & 79 & 0.87 \\
\hline Benefits & 153 & 95 & 78 & 0.85 \\
\hline Transparency & 142 & 97 & 84 & 0.9 \\
\hline Flexibility & 137 & 97 & 87 & 0.91 \\
\hline Environment & 130 & 92 & 79 & 0.85 \\
\hline D\&I & 126 & 95 & 72 & 0.82 \\
\hline Overhead & 115 & 97 & 81 & 0.88 \\
\hline Initiatives & 112 & 94 & 85 & 0.89 \\
\hline Retention & 89 & 90 & 73 & 0.8 \\
\hline Accountability & 79 & 91 & 76 & 0.82 \\
\hline Regional & 50 & 94 & 53 & 0.7 \\
\hline Telework & 50 & 98 & 81 & 0.89 \\
\hline
\end{tabular}

TAB LE 2 Per-code model performance summary. N Training indicates the number of positive examples in the training data.

responses, open coding, focused coding, refining codes, etc.). Members of the coding team reported the time spent reviewing responses was reduced given that predictions were already made about which codes should likely be applied to each response. This could be a result of the task coders were completing required less cognitive overhead—that is, determining whether a prediction was correct is easier than determining which of 24 codes needed to be applied.

While human consistency (e.g., inter-rater reliability) was not directly measured during the initial human coding that resulted in the training dataset, inter-rater reliability is always a concern when dealing with human coders. Another benefit of the model is the consistency in outputting the same predictions across the entirety of the dataset. In short, many of the issues researchers often encounter when manually coding, including human fatigue, variability in interpretation, intercoder reliability (IRR), and data entry errors are mitigated when using the model. 


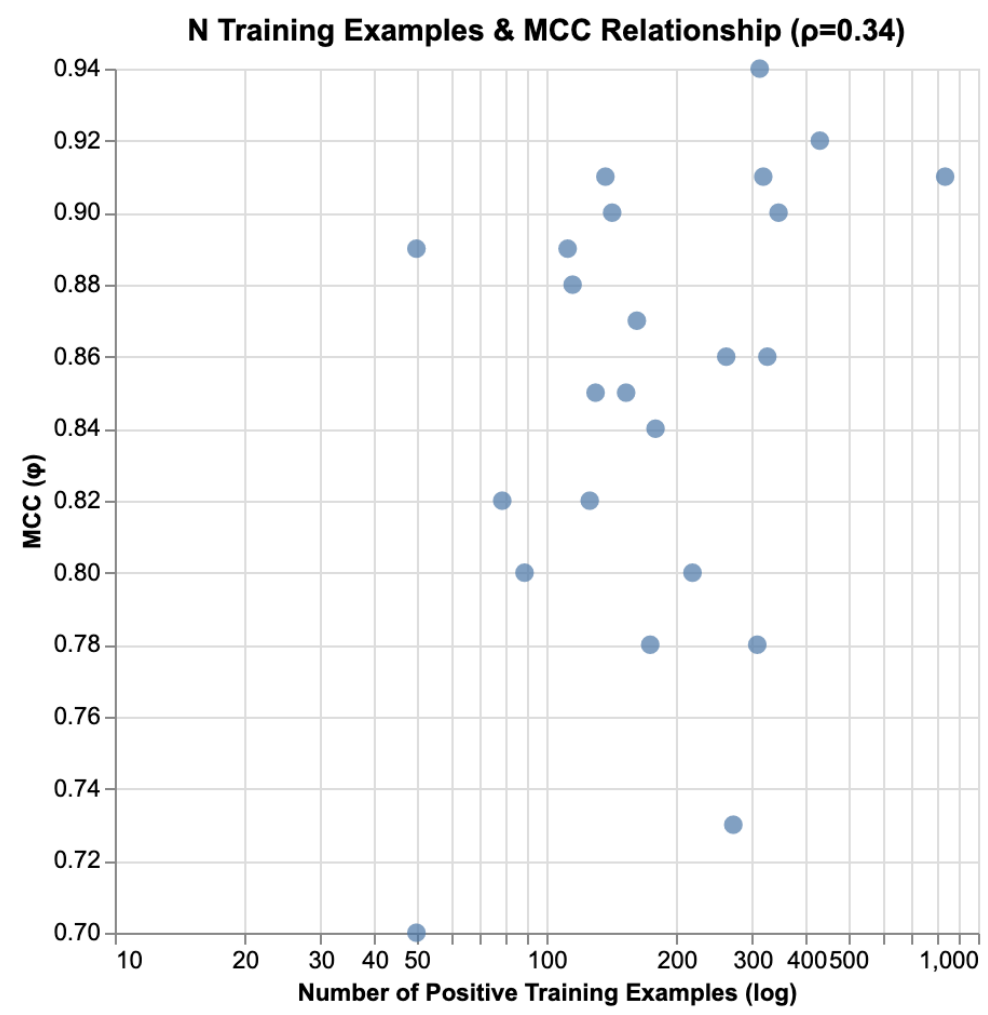

FIG URE 3 Relationship and correlation between MCC and number of training examples. Note the $\mathrm{x}$-axis is $\log 10 \mathrm{scaled}$ and the $y$-axis starts at 0.7 .

This method appears to be faster and take less time than "traditional" coding approaches. The potential for improved speed of coding allows for data to be acted on sooner. For example, in this case findings from open-ended items were more quickly passed to leadership, who in turn were able to more quickly plan for possible actions based on the results. The amount of time it takes to analyze qualitative data is often a contributing factor for data not being fully utilized-this method could reduce time spent coding the data, which in turn frees up researchers' time to better understand and interpret findings (e.g., understanding the relationships between codes, developing larger themes etc.).

\section{| Limitations}

While successfully predicting the full code set achieved a rate of $67.5 \%$ for all responses $(81.5 \%$ of the individual code applications), there are limitations to this approach. First, this process required enough manually coded data for model training. That is, a previously constructed set of codes had to be developed and then applied to responses by the team using the traditional qualitative coding methods using human coders.

In practice, the model can only predict codes that exist in the training data with enough examples of coded responses. There is a weak relationship $(\rho=0.34)$ between the number of positive training examples and the performance (MCC) of the model. If there are code(s) that are particularly important to the research question, but are not well represented in the training data, they 
might not be good candidates for prediction without the inclusion of additional training examples. After all, the sheer number of codes (or lack thereof) may not be indicative of the importance of the finding. In addition, for codes that have a low tolerance for error, automation or prediction is not likely to be the correct choice for researchers. For example, one of the topics included in our original coding scheme was Ethics Issues-however, we did not automate the prediction of this code because there is no tolerance for a false negative as all ethical issues mentioned in the responses need to be identified without error and coded correctly.

Related to the limitation above, this model does not eliminate the need for researchers to review data from subsequent collections (e.g., data collected at a different point in time as the training dataset) as the model is unable to predict new codes. Even if data are from the same survey question, responses still need to be reviewed to determine if additional codes are needed to classify new topics/concepts being observed in the data that were not present in the original training dataset.

\section{| Future Work \& Potential Use Cases}

There are several additional applications of a model that predicts codes that we did not evaluate. Since we were able to capture a relatively high proportion of the final codes in prediction, and we know the model performance per class, the predicted codes could be used to deliver preliminary results of the coding, with the caveat that some proportion coded responses are absent.

There are additional model outputs that could also be used for coding related tasks. First, each response has a predicted probability of being assigned to each code. These probabilities could be used in a multitude of ways. The collection of code probabilities per response could be combined to identify new topics that are a combination of existing topics, based on the distribution of probabilities across all codes. The probabilities could also be used to deliberately select a cutoff for code assignment. For example, if the tolerance for false negatives on the Transparency code is low, the threshold could be bumped up such that any response with a probability over 0.25 , rather than 0.5 , was assigned that code from the model.

One exciting use case is combining a model-based approach with a human-based approach to improve the whole qualitative analysis process. There are insights to be gained in understanding why a human and a model might make different assignments: is the idea too abstract? Did the training data have low IRR? Are there unique language attributes of certain responses only identifiable by humans? A fluid "conversation" back and forth between an analyst and a model could provide novel ideas for the coding scheme or analysis process.

One large, unexplored avenue of possibility would be to use the embeddings generated in the model training process to better understand how the model itself is grouping and organizing responses by similarity. We did not take advantage of models specifically trained to generate embeddings according to similarity, which could provide possibilities for cluster-based coding schemes that complement existing human-created schemes.

\section{CONCLUSION}

Qualitative analysis, machine learning, and surveys are three concepts that have been in each other's orbit for quite some time. With the advent of transfer learning, which enables accurate performance on prediction given sample sizes typically present in surveys, the connection between these fields grows stronger.

We demonstrated the use of BERT, a transfer learning architecture, to predict the application of 24 codes to almost 3000 responses to an internal employee survey. The model achieved satisfactory performance when evaluated after a team of humans had adjudicated the predicted codes. As a result, the time spent on qualitative data analysis was significantly reduced, allowing findings to be acted on sooner than they would have been otherwise. This is particularly important, as the time it takes to properly analyze qualitative data is one of the primary reasons open-ended survey items are often avoided, or if they are used, the resulting data under-utilized. 
This paper demonstrates this particular methodology could be applied to other surveys that repeatedly collect data on the same open-ended question: whether that's a longitudinal survey or a long-running survey with several thousand responses needing to be coded.

\section{APPENDIX}

\section{REFERENCES}

Omar Abdelwahab, Mohamed Bahgat, Christopher J. Lowrance, and Adel Elmaghraby. Effect of training set size on SVM and Naïve Bayes for Twitter sentiment analysis. In 2015 IEEE International Symposium on Signal Processing and Information Technology (ISSPIT). IEEE, dec 2015. doi: 10.1109/isspit.2015.7394379. URL https://doi.org/10.1109\%2Fisspit.2015.7394379.

Virginia Braun and Victoria Clarke. Using thematic analysis in psychology. Qual. Res. Psychol., 3(2):77-101, jan 2006.

Kathy Charmaz. Constructing Grounded Theory: A Practical Guide through Qualitative Analysis. SAGE, jan 2006.

Davide Chicco and Giuseppe Jurman. The advantages of the Matthews correlation coefficient (MCC) over F1 score and accuracy in binary classification evaluation. BMC genomics, 21(1):1-13, 2020.

Juliet M Corbin and Anselm Strauss. Grounded theory research: Procedures, canons, and evaluative criteria. Qual. Sociol., 13(1):3-21, mar 1990.

Kevin Crowston, Xiaozhong Liu, and Eileen E Allen. Machine learning and rule-based automated coding of qualitative data. proceedings of the American Society for Information Science and Technology, 47(1):1-2, 2010.

Martyn Denscombe. Item non-response rates: a comparison of online and paper questionnaires. Int. J. Soc. Res. Methodol., 12(4): 281-291, oct 2009 .

Jacob Devlin, Ming-Wei Chang, Kenton Lee, and Kristina Toutanova. Bert: Pre-training of deep bidirectional transformers for language understanding. arXiv preprint arXiv:1810.04805, 2018.

Peter Flach. Machine Learning: The Art and Science of Algorithms that Make Sense of Data. Cambridge University Press, sep 2012.

Peter A Flach and Meelis Kull. Precision-Recall-Gain curves: PR analysis done right. In Proceedings of the 28th International Conference on Neural Information Processing Systems-Volume 1, pages 838-846, 2015.

Jeremy Howard and Sebastian Ruder. Universal language model fine-tuning for text classification. arXiv preprint arXiv:1801.06146, 2018.

R Janani and S Vijayarani. Text Classification: A Comparative Analysis of Word Embedding Algorithms. International Journal of Computer Sciences and Engineering, 7(4):818-822, 2019.

Paul F. Lazarsfeld. The Controversy Over Detailed Interviews-An Offer for Negotiation. Public Opinion Quarterly, 8(1):38, 1944. doi: 10.1086/265666. URL https://doi.org/10.1086\%2F265666.

Tomas Mikolov, Ilya Sutskever, Kai Chen, Corrado Greg S, and Jeff Dean. Distributed Representations of Words and Phrases and their Compositionality. In C J C Burges, L Bottou, M Welling, Z Ghahramani, and K Q Weinberger, editors, Advances in Neural Information Processing Systems 26, pages 3111-3119. Curran Associates, Inc., 2013.

M B Miles, A M Huberman, and J Saldaña. Qualitative Data Analysis: A Methods Sourcebook. SAGE Publications, 2013.

Urša Reja, Katja Lozar Manfreda, Valentina Hlebec, and Vasja Vehovar. Open-ended vs. close-ended questions in web questionnaires. Developments in applied statistics, 19(1):159-177, 2003.

Philip J Stone, Dexter C Dunphy, and Marshall S Smith. The general inquirer: A computer approach to content analysis. MIT press, 1966. 


\begin{tabular}{|c|c|c|c|c|c|c|c|c|c|}
\hline & $\begin{array}{l}\text { Preci- } \\
\text { sion }\end{array}$ & $\begin{array}{l}\mathrm{Re}- \\
\text { call }\end{array}$ & $\mathrm{MCC}$ & $\begin{array}{c}\mathrm{N} \\
\text { Total }\end{array}$ & $\begin{array}{c}\mathrm{N} \\
\text { Predicted }\end{array}$ & $\begin{array}{c}\% \\
\text { Total }\end{array}$ & $\begin{array}{c}\% \\
\text { Predicted }\end{array}$ & $\begin{array}{c}\mathrm{N} \\
\text { Training }\end{array}$ & $\begin{array}{c}\% \\
\text { Training }\end{array}$ \\
\hline \multicolumn{10}{|l|}{ Code } \\
\hline Development & 0.97 & 0.91 & 0.91 & 1009 & 950 & 0.35 & 0.33 & 839 & 0.29 \\
\hline Management & 0.96 & 0.9 & 0.92 & 459 & 428 & 0.16 & 0.15 & 430 & 0.15 \\
\hline Processes & 0.98 & 0.85 & 0.9 & 374 & 326 & 0.13 & 0.11 & 345 & 0.12 \\
\hline $\begin{array}{l}\text { Communica- } \\
\text { tion }\end{array}$ & 0.96 & 0.8 & 0.86 & 350 & 292 & 0.12 & 0.1 & 325 & 0.11 \\
\hline Teamwork & 0.95 & 0.89 & 0.91 & 266 & 248 & 0.09 & 0.08 & 318 & 0.11 \\
\hline Fairness & 0.96 & 0.66 & 0.78 & 324 & 221 & 0.11 & 0.08 & 308 & 0.11 \\
\hline Compensation & 0.96 & 0.94 & 0.94 & 362 & 354 & 0.12 & 0.12 & 312 & 0.11 \\
\hline Strategy & 0.97 & 0.58 & 0.73 & 289 & 175 & 0.1 & 0.06 & 271 & 0.09 \\
\hline International & 0.94 & 0.81 & 0.86 & 290 & 250 & 0.1 & 0.09 & 261 & 0.09 \\
\hline Workload & 0.93 & 0.71 & 0.8 & 245 & 186 & 0.08 & 0.06 & 218 & 0.08 \\
\hline Recognition & 0.88 & 0.82 & 0.84 & 179 & 168 & 0.06 & 0.06 & 179 & 0.06 \\
\hline Satisfied & 0.94 & 0.66 & 0.78 & 176 & 125 & 0.06 & 0.04 & 174 & 0.06 \\
\hline Morale & 0.97 & 0.79 & 0.87 & 175 & 144 & 0.06 & 0.05 & 162 & 0.06 \\
\hline Benefits & 0.95 & 0.78 & 0.85 & 224 & 183 & 0.08 & 0.06 & 153 & 0.05 \\
\hline Transparency & 0.97 & 0.84 & 0.9 & 135 & 117 & 0.05 & 0.04 & 142 & 0.05 \\
\hline Flexibility & 0.97 & 0.87 & 0.91 & 159 & 144 & 0.05 & 0.05 & 137 & 0.05 \\
\hline Environment & 0.92 & 0.79 & 0.85 & 72 & 62 & 0.02 & 0.02 & 130 & 0.05 \\
\hline D\&I & 0.95 & 0.72 & 0.82 & 119 & 91 & 0.04 & 0.03 & 126 & 0.04 \\
\hline Overhead & 0.97 & 0.81 & 0.88 & 162 & 136 & 0.06 & 0.05 & 115 & 0.04 \\
\hline Initiatives & 0.94 & 0.85 & 0.89 & 55 & 50 & 0.02 & 0.02 & 112 & 0.04 \\
\hline Retention & 0.9 & 0.73 & 0.8 & 99 & 80 & 0.03 & 0.03 & 89 & 0.03 \\
\hline $\begin{array}{l}\text { Accountabil- } \\
\text { ity }\end{array}$ & 0.91 & 0.76 & 0.82 & 107 & 89 & 0.04 & 0.03 & 79 & 0.03 \\
\hline Regional & 0.94 & 0.53 & 0.7 & 64 & 36 & 0.02 & 0.01 & 50 & 0.02 \\
\hline Telework & 0.98 & 0.81 & 0.89 & 67 & 55 & 0.02 & 0.02 & 50 & 0.02 \\
\hline
\end{tabular}

TA B LE 3 Full Code Information. N Total is the number of positive examples after adjudication, N Predicted is the Number Predicted, \% Total is the percent of responses that had that code after adjudication, \% Predicted is the percent of responses predicted to have that code, $\mathrm{N}$ Training is the number of positive examples in the training dataset, and \% Training is the percent of training data responses assigned that code.

342 Phillip Stone and Roger Hurwitz. Inquirer Home Page. http://www.wjh.harvard. edu/ inquirer/, 2012. Accessed: 2020-4-9.

343 Roger Tourangeau, Lance J Rips, and Kenneth Rasinski. The Psychology of Survey Response. Cambridge University Press, mar 2000. 
Abinash Tripathy, Ankit Agrawal, and Santanu Kumar Rath. Classification of sentiment reviews using n-gram machine learning approach. Expert Syst. Appl., 57:117-126, sep 2016.

Gregor Wiedemann, Steffen Remus, Avi Chawla, and Chris Biemann. Does BERT make any sense? Interpretable word sense disambiguation with contextualized embeddings. arXiv preprint arXiv:1909.10430, 2019. 